\title{
The Frequency of Epidermal Growth Factor Receptor (EGFR) mutations in Iraqi patients with Non-Small Cell Lung Cancer (NSCLC)
}

\author{
Hanan H Ramadhan ${ }^{1 *}$, Dhuha F Taaban ${ }^{1}$, Jubran K Hassan²
}

\begin{abstract}
Objective: Non-Small Cell Lung Cancer (NSCLC) Carcinogenesis could be caused by numerous genetic mutations, one of the most common is the mutation in the Epidermal Growth Factor Receptor $(E G F R)$ which was used in the advanced stages of the disease as a therapeutic goal. This study aims to estimate the frequency of Epidermal Growth Factor Receptor mutations in Iraqi patients with Non-Small Cell Lung Cancer. Methods: One hundred thirty-eight patients confirmed with NSCLC have participated in this study, patients were sent for EGFR testing by different oncology centers in Iraq. Data and samples were collected. The Mutation was detected using COBAS ${ }^{\circledR}$ DNA Sample Preparation Kit that designed to detect the following mutations: Exon 19: deletions and complex mutations; Exon 21: L861Q and L858R; Exon 18 mutation: G719X (G719A, G719C, and G719S); Exon 20: S768I, T790M, and insertions, this kit utilizes the technology of the real time Polymerase Chain Reaction. Results: This study was included 79 males and 59 females, with a mean age of $60.1 \pm 12.4$ years. A positive EGFR mutations were found in $38(27.53 \%)$ of samples. Exon 19 deletions (25/38, 65.8\%) and substitution L858R in exon 21 10/38 (26.3\%) were the most common mutations. Multiple mutations (Exon 20 and 19 combined together) were founded in 2/38 (5.3\%), and 1/38 (2.6\%) $A L K$ mutation. Non-significant differences among age groups and gender in the incidence of mutations were found. Conclusion: The current study represents the first epidemiological study in Iraq to find EGFR mutations frequency among NSCLC patients that reveals the incidence rate of $27.53 \%$, which is between the higher prevalence rate in Asian populations and lower rates in western countries. These results explain the genetic differences of NSCLC in the world due to ethnic differences of the population, more studies are needed in Arab countries to study the EGFR mutations, find the effect of ethnicity and environmental factors for lung cancer, and the subsequent therapy.
\end{abstract}

Keywords: Non - small cell lung cancer (NSCLC)- epidermal growth factor receptor mutations

Asian Pac J Cancer Prev, 22 (2), 591-596

\section{Introduction}

Lung cancer is the most probable cause of cancer incidence and mortality in the world, with more than 2 million new cases and 1.8 million deaths in 2018 (Bray et al., 2018). Non-small cell lung cancer (NSCLC) accounts for $85 \%$ of overall lung cancers (Zhang et al., 2016). Unfortunately $70 \%$ of NSCLC patients are diagnosed at an advanced stage, therefore they are not competent for the surgery (Zhou et al., 2017). The treatment protocol based on Chemotherapy as the first choice in the treatment (Goldstraw et al., 2011). Although the overall survival was prolonged and the quality of life was improved by chemotherapy, the prognosis is still poor, particularly in advanced NSCLC (Miltiadou et al., 2020). The median overall survival is 1 year and only $3.5 \%$ of patient will survive 5 years after diagnosis(Cataldo et al., 2011). In 2013, a meta-analysis had founded that, the carcinogenesis in NSCLC can be caused by different mutations, one of the most frequent is the mutation in the epidermal growth factor receptor (EGFR) (Dearden et al., 2013). The activation of EGFR achieved by binding of its ligands, somatic mutations in these receptors will cause uncontrolled cell division and proliferation by constant receptor activation (Izadian et al., 2017; Benbrahim et al., 2018) .

The EGFR mutations consider a potential therapeutic target, which is more frequent in adenocarcinomas (ADC) patients and less common in squamous cell carcinoma (SCC) (Yatabe et al., 2015). These mutations are more common among women and non-smokers (Kota et al., 2015). The EGFR mutation rates have been variable according to ethinicty with higher frequency in Asian as compared with western lung cancer patients (Midha et al., 2015; Yatabe et al., 2015). The EGFR mutations frequency in Asians range from $27 \%$ to $60 \%$ while in 
European $8 \%$ to $13 \%$, the frequency in Africans reaches to $12 \%, 16 \%$ reported in white Americans, and $20 \%$ to $25 \%$ in India (Palacio et al., 2019). Unfortunately, there are a limited data regarding EGFR mutations in the middle east (Benbrahim et al., 2018), with the absence of frequency determination in Iraq.

In lung cancer, the deletion mutation in exon 19 and point mutation in exon 21 (L858R) represent $90 \%$ of EGFR mutations. whereas the T790M mutations are of low frequency (Arrieta et al., 2015). The active EGFR mutations in NSCLC patients exhibit Progression-Free Survival (PFS) to be longer and Objective Remission Rate (ORR) would be better by using EGFR Tyrosine Kinase Inhibitors (TKI) therapy, it also improves life quality, and decrease the side effects related to the treatment when compared with chemotherapy receiving patients (Yatabe et al., 2015). In NSCLC patients several guidelines strongly recommended the testing for $E G F R$ mutations and suggested early EGFR-TKIs therapy in patients harboring it (Zhou et al., 2017). Thus, there is a great interest to determine the frequency of EGFR mutations. This study aims to estimate the frequency of $E G F R$ mutations in Iraq.

\section{Materials and Methods}

\section{Patients}

This retrospective study included 158 participants with confirmed NSCLC, who have been referred for EGFR testing from different oncology centers throughout Iraq between September 2018 and February 2019. Written informed consent was supplied to all participated members in the current study. The ethics committee in the college of pharmacy/ University of Basrah approved this study. About 20 samples were excluded because of the small sample size, the remaining is 138 samples. Patients' Demographic data include age, gender, and smoking habits were recorded. No other pathological or clinical information was available since the samples were collected from different cities in the country for this analysis.

Samples were obtained by small Tru-cut biopsy, and the isolated DNA was obtained from the Formalin Fixed Paraffin Embedded (FFPE) tissues.

\section{Extraction of DNA and Mutational analysis}

Biopsies were collected by Tru-cut biopsy, then the paraffin embedded sections were prepared for DNA extraction process. The DNA Sample Preparation Kit from $\operatorname{cobas}^{\circledR}$, is used for DNA extraction from $(5-\mu \mathrm{m})$ section of formalin fixed paraffin embedded tumors (FFPET) specimens, by preparing the specimen manually depend on the binding of nucleic acid to the glass fibers. The amount of DNA is determined by spectrophotometer, and then a certain concentration was adjusted to be used in the amplification/detection mixture. The cobas z 480 analyzer amplified and detected the target DNA in cobas ${ }^{\circledR}$ EGFR Test using already provided reagents for the amplification - detection. The cobas ${ }^{\circledR}$ EGFR Test is used to perform the EGFR mutational analysis, The following mutations can be detected by this kit: Exon 19: deletions and complex mutations; Exon 21: L861Q and L858R;
Exon 18 mutation: $G 719 X(G 719 A, G 719 C$, and G719S); Exon 20: S768I, T790M, and insertions, this kit utilizes the technology of the real time Polymerase Chain Reaction (PCR), the target DNA amplification and detection by PCR using fluorescent dyes labeled complementary primer pairs and oligonucleotide probes.

\section{Statistical analysis}

The data were statistically analyzed by using statistical package for social sciences (SPSS) version 19.0 for windows software. The results of the study are expressed as the mean \pm standard deviation (SD), The differences between groups were performed by using the chi-square test. The statistical significance was considered at a p-value of less than 0.05 .

\section{Results}

The population of the study consists of 158 tumor samples, which were received for analysis of $E G F R$ mutations, 20 samples with a small sample size were excluded. In all patients, the most common EGFR mutations (exon 19 deletion, exon 20 T790M mutation, and exon $21 \mathrm{~L} 858 \mathrm{R}$ point mutation) were analyzed. Out of 138 studied cases, $79(57.2 \%)$ were males and $59(42.8 \%)$ were female (Table 1$)$. The mean age of the participant was $60.1 \pm 12.4$ years. A positive $E G F R$ mutations were detected in 38 patients $(27.53 \%)$, the most frequent mutation was a deletion in exon 19 (25 of 38 patients, $65.8 \%)$, while only 10 of $38(26.3 \%)$ patients had a substitution L858R in exon 21. Multiple mutations (Exon 19 and Exon 20 mutations combined together) have been found in 2 of 38 patients (5.3\%), table 1 shows the distribution of EGFR mutations. Only 1 case showed another type of mutation, ALK mutation (2.6\%). There was no evidence of mutations that could be found in 100 patients $(72.47 \%)$ (Table 1$)$.

Table 2 showed that EGFR mutations were detected in 16 of 59 females (27.1\%), and 22 of 79 males (27.8\%),

Table 1. Demographic Data of Patients

\begin{tabular}{lcc}
\hline & study group N=138 & p- value \\
\hline Age ( years) & $60.1 \pm 12.4$ & 0.562 \\
$35-50$ & $37(26.8 \%)$ & 0.009 \\
$50-65$ & $38(27.5 \%)$ & \\
$>65$ & $63(45.7 \%)$ & \\
Gender & & \\
Male & $79(57.2 \%)$ & 0.0887 \\
Female & $59(42.8 \%)$ & \\
$\%$ Mutation & & \\
+ve mutation & $38(27.53 \%)$ & $<0.0001$ \\
-ve mutation & $100(72.47 \%)$ & \\
Mutation distribution N=38 & & \\
L858R & $10(26.3 \%)$ & $<0.0001$ \\
Ex19Del & $25(65.8 \%)$ & \\
Ex19Del, T790M & $2(5.3 \%)$ & \\
ALK Mutation & $1(2.6 \%)$ & \\
\hline p value $<0.05$ considered as significant values &
\end{tabular}

$\mathrm{p}$ value $<0.05$ considered as significant values 
Prevelence of mutations

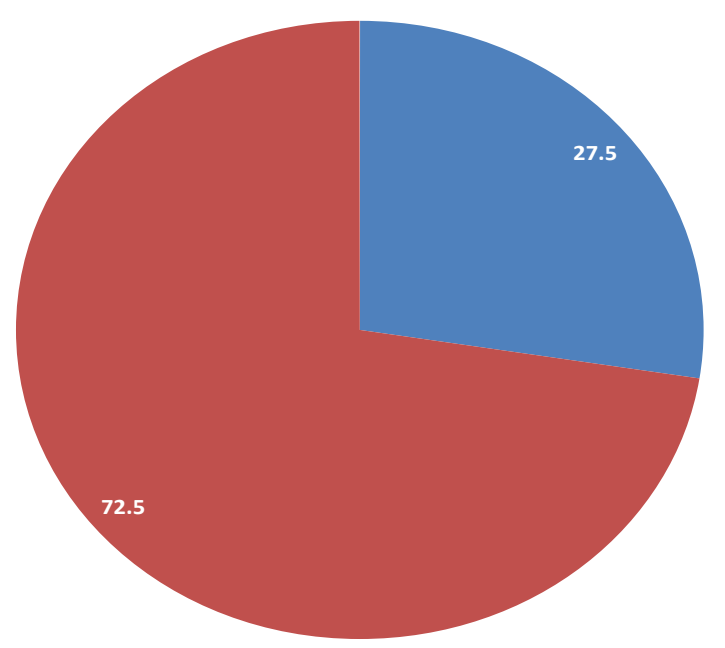

- Positive Muation negative mutation

Figure 1. The Prevalence of EGFR Mutations in Iraq.

with no statistically significant differences, have been found between genders ( $\mathrm{p}=0.924)$, among age groups. $(p=0.816)$ and different regions in Iraq $(p=0.892)$.

A subgroup analysis was also conducted to detect whether the distribution of EGFR mutation types was influenced by gender or age. This study revealed that there were no significant differences were observed in the distribution of types of EGFR mutation by sex and age. As seen in (Tables 3 and 4).

\section{Mutation types}

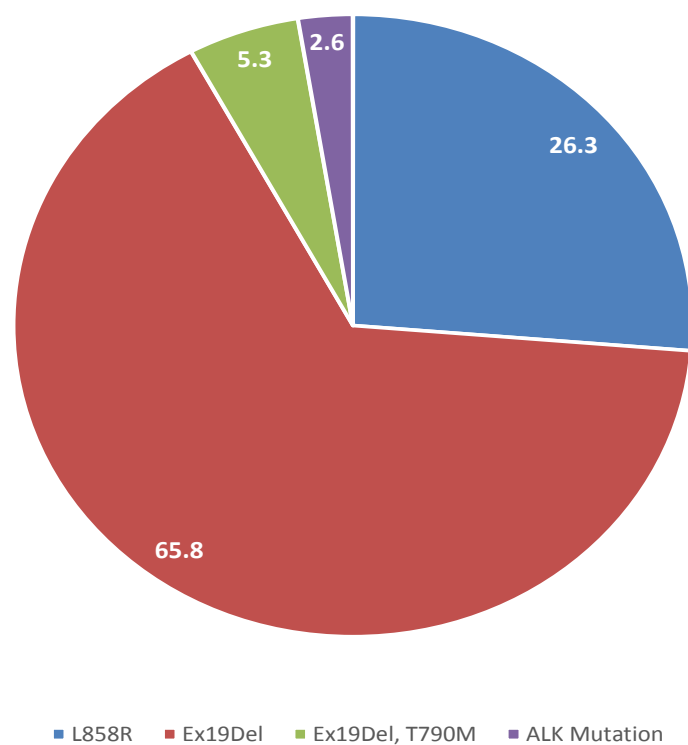

Figure 2. The Distribution of Mutations by Exon.

\section{Discussion}

The analysis of EGFR mutations is important for the diagnosis and treatment decision in NSCLC, especially adenocarcinoma, as EGFR mutations are a potential predictive biomarker for the EGFR-TKI therapy response (Zhou and Christiani, 2011; Shi et al., 2015). The overall prognosis can be improved by EGFR mutations testing and using the EGFR-TKI therapy early, as the chemotherapy has limited efficacy in NSCLC patients (Zhang et al., 2016). In the present study, 138 patient samples from different cities were analyzed to estimate the EGFR

Table 2. Mutation Prevalence According to Gender and Age

\begin{tabular}{|c|c|c|c|c|}
\hline Parameter & Total & Positive & Negative & P values \\
\hline \multicolumn{5}{|c|}{ Mutation prevalence according Gender } \\
\hline Male & 79 & $22(27.8 \%)$ & $57(72.2 \%)$ & 0.924 \\
\hline Female & 59 & $16(27.1 \%)$ & $43(72.9 \%)$ & \\
\hline \multicolumn{5}{|c|}{ Mutation prevalence according Age } \\
\hline $35-50$ & 37 & $11(29.7 \%)$ & $26(70.3 \%)$ & 0.816 \\
\hline $50-65$ & 38 & $9(23.7 \%)$ & $29(76.3 \%)$ & \\
\hline$\geq 65$ & 63 & $18(47.4 \%)$ & & \\
\hline \multicolumn{5}{|c|}{ Mutation prevalence according to Regions } \\
\hline Babil & 11 & $4(36.4 \%)$ & $7(63.6 \%)$ & 0.892 \\
\hline Baghdad & 44 & $11(25 \%)$ & $33(75 \%)$ & \\
\hline Basra & 21 & $7(33.3 \%)$ & $14(66.7 \%)$ & \\
\hline AL-Diwania & 4 & $1(25 \%)$ & $3(75 \%)$ & \\
\hline Erbil & 4 & $2(50 \%)$ & $2(50 \%)$ & \\
\hline Kirkuk & 7 & $3(42.9 \%)$ & $4(57.1 \%)$ & \\
\hline Karbala & 9 & $3(33.3 \%)$ & $6(66.7 \%)$ & \\
\hline Najaf & 8 & $1(12.5 \%)$ & $7(87.5 \%)$ & \\
\hline Sulaimanyah & 13 & $2(15.4 \%)$ & $11(84.6 \%)$ & \\
\hline Thi-Qar & 10 & $4(40 \%)$ & $6(60 \%)$ & \\
\hline
\end{tabular}


Table 3. Positive Mutation Details According to Gender

\begin{tabular}{lccc}
\hline Parameter & \multicolumn{2}{c}{ Gender } & P values \\
& Male & female & \\
\hline L858R & $6(27.3 \%)$ & $4(25 \%)$ & 0.877 \\
Ex19Del & $13(59.1 \%)$ & $12(75 \%)$ & 0.314 \\
Ex19Del, T790M & $2(9.1 \%)$ & $0(0 \%)$ & 0.221 \\
ALK Mutation & $1(4.5 \%)$ & $0(0 \%)$ & 0.394 \\
\hline p
\end{tabular}

$\mathrm{p}$ value $<0.05$ considered as significant values

mutations frequency in Iraq. This is the first study in Iraq, so the results can be used as a reference for research in the future.

In the current study, the EGFR mutations were detected in $27.53 \%$ of NSCLC patients. Despite, the prevalence rate was higher than that reported in the Saudi population and Lebanese population (Al-Kuraya et al., 2006; Fakhruddin et al., 2014; Kattan et al., 2015; Naderi et al., 2015), and higher than the prevalence rate reported by Tfayli et al in 2017 published study (Tfayli et al., 2017), this finding was still consistent with EGFR mutations frequency in the Middle East region, that ranging from 2.9\% to 28.7\% (Errihani et al., 2013; Jazieh et al., 2015; Zaki et al., 2015; Benbrahim et al., 2018), and lower than Asian countries that reached $45.5 \%$ (Yatabe et al., 2015; Tfayli et al., 2019). However, a review of 19 European studies by Szumera-Cieckiewicz et al, reported frequencies from European countries between $(2.6 \%$ and $39 \%)$ in Italy and Germany, respectively (Szumera-Cieckiewicz et al., 2013). In Latin America, the updated research of 5738 cases in 2015, revealed that the frequency of EGFR mutations varies from $14.4 \%$ in Argentina, to $51.1 \%$ in Peru (Arrieta et al., 2015). Leary et al, 2012, revealed the incidence of EGFR mutations in UK was $11 \%$ (Leary et al., 2012). Another study from turkey demonstrated a frequency of EGFR mutations was 30\% (A et al., 2018). Similarly, a study in the US, conducted that the EGFR mutations prevalence rate was $20 \%$ (Reinersman et al., 2011), and Michele L. Cote et al reported the frequency of $13.9 \%$ in African-Americans (Cote et al., 2011). These differences in prevalence rate have resulted from different geographical regions, the ethnicity of these populations (Arrieta et al., 2015; Zhou et al., 2017; Benbrahim et al., 2018), and also may be due to differences in the testing methods (Benbrahim et al., 2018).

In the current study, deletion in exon 19 was the most frequent type of EGFR mutations (65.8\%) followed by L $858 \mathrm{R}(26.3 \%)$ of all mutations, this is similar to studies in the East Asian countries (Liam et al., 2013; Shi et al., 2014; Lee et al., 2015; Shi et al., 2015). And is comparable to the study published in 2017 , that revealed a deletion in exon 19 represents $78.1 \%$, followed by exon 21 mutation $21.9 \%$ of all mutations (Tfayli et al., 2017). In contrast, Berois et al, reports that the most common mutations are rare mutations and only $40 \%$ of all mutations is the mutations in exon 19 and exon 21 (Berois et al., 2017). Liu et al, reported that (80-90\%) of all mutations are the deletion mutation in exon 19 and missense mutation L858R in exon 21 together, these would be the best predictors for TKIs response, with
Table 4. Positive Mutation Details According to Age

\begin{tabular}{lcccc}
\hline Type of Mutation & $\begin{array}{c}35-50 \\
\text { years } \\
\mathrm{N}=11\end{array}$ & $\begin{array}{c}50-65 \\
\text { years } \\
\mathrm{N}=9\end{array}$ & $\begin{array}{c}\geq 65 \text { years } \\
\mathrm{N}=18\end{array}$ & $\begin{array}{c}\mathrm{P} \\
\text { values }\end{array}$ \\
\hline L858R & $4(36.4 \%)$ & $2(22.2 \%)$ & $4(22.2 \%)$ & 0.668 \\
Ex19Del & $6(54.5 \%)$ & $6(66.7 \%)$ & $13(72.2 \%)$ & 0.621 \\
Ex19Del, T790M & $1(9.1 \%)$ & $0(0 \%)$ & $1(5.6 \%)$ & 0.661 \\
ALK Mutation & $0(0 \%)$ & $1(11.1 \%)$ & $0(0 \%)$ & 0.214 \\
P value & 0.1778 & 0.097 & 0.0015 & \\
\hline p value $<0.05$ considered as significant values & &
\end{tabular}

better outcomes reported for exon 19 deletions than point mutation L858R (Liu et al., 2016). Whereas, the T790M mutation found as a complex mutation in 2 patients $(5.3 \%)$ of all mutations. However, this result is higher than the incidence of T790M mutation reported by Zhou et al, and Shi et al (Shi et al., 2015; Zhou et al., 2017). These differences in the prevalence of T790M EGFR mutation may be due to variations in the technique and population (Zhou et al., 2017).

The response to treatment, prognosis, and survival rates varies with the mutation type, so the study of mutations type is of particular clinical importance. These findings have been supported by several studies, Jackman et al., 2006 and Li at al., 2011 who reported that better prognosis and long survival were associated with the mutation in exon 19 and 21, whereas poor prognosis was correlated with a mutation in exon 20 (Jackman et al., 2006; Li et al., 2011). Accordingly response rate to the treatment with TKIs were found to be improved in exon 19 and 21 mutations while exon 20 was showed resistance to the therapy (Unal et al., 2013). Longer survival rates were detected in patients with exon 19 deletions treated with TKIs (Cote et al., 2011), while short progression free survival has been exhibited in NSCLC patient with T790M mutation received EGFR-TKI treatment (Shi et al., 2015).

The previous Asian studies conducted a higher prevalence rate of EGFR mutations in the female gender. Leary et al., (2012) and Demiray et al., (2018) are in line with the Asian results and consider the female gender as one of the predictors of EGFR mutations (Leary et al., 2012; A et al., 2018). Another study published in 2015, from three countries KSA, United Arab Emirates and Qatar demonstrated the significant association of the EGFR prevalence rate with the female gender (Jazieh et al., 2015). Interestingly, in our study, the EGFR mutations were found in $27.1 \%$ females and $27.8 \%$ male patients, and the effect of gender was not significant. This result is in agreement with the published study by Unal et al, who found that, although the EGFR mutation rate is higher in females, it does not reach a statistically significant level (Unal et al., 2013).

This study did not find any significant difference in the rate of mutations across different regions in Iraq, that have been suggested, the highest EGFR mutations rate was in Baghdad 13 (34.2\%), followed by Basra 7 (18.4), and the lowest rate was in Erbil and Al-Diwania 1(2.6\%). A larger scale study with an extended period of time required to study the differences in mutation rate among different 
geographic regions.

\section{Acknowledgements}

Authors would like to express their appreciation to University of Basrah for its attention and continuous encouragement during this work.

\section{Funding}

This research received no specific grant from funding agencies in the public, commercial, or not-for-profit sectors. The research was approved by college of pharmacy/ university of Basrah.

The research is not a part of an approved thesis.

\section{Ethical approval}

The present study has been done after approved by Research Ethics Committee of pharmacy College, University of Basrah.

\section{Author contributions}

Hanan H. Ramadhan: Conception and design of study, collecting the data, writing the paper, and review it. Dhuha F. Taaban: participating in the collecting of information in many references, and writing it. Jubran K. Hassan : data analysis, review the paper and editing. This authorship contribution statement is signed by the corresponding author on behalf of all the listed authors in the manuscript.

\section{Conflict of interest}

authors reported no conflicts of interest.

\section{References}

A D, A Y, N K, et al (2018). The frequency of EGFR and KRAS mutations in the Turkish population with non-small cell lung cancer and their response to Erlotinib therapy. Balkan JMed Genet, 21, 21-6.

Al-Kuraya K, Siraj AK, Bavi P, et al (2006). High epidermal growth factor receptor amplification rate but low mutation frequency in Middle East lung cancer population. Hum Pathol, 37, 453-7.

Arrieta O, Cardona AF, Martin C, et al (2015). Updated frequency of EGFR and KRAS mutations in non smallcell lung cancer in Latin America: The Latin-American Consortium for the Investigation of Lung Cancer (CLICaP). $J$ Thorac Oncol, 10, 838-43.

Benbrahim Z, Antonia T, Mellas N (2018). EGFR mutation frequency in Middle East and African non-small cell lung cancer patients: a systematic review and meta-analysis. $B M C$ Cancer, 18, 891.

Berois N, Touya D, Ubillos L (2017). Prevalence of EGFR mutations in lung cancer in Uruguayan population. J Cancer Epidemiol, 2017, 6170290.

Bray F, Ferlay J, Soerjomataram I, et al (2018). Global cancer statistics 2018: GLOBOCAN estimates of incidence and mortality worldwide for 36 cancers in 185 countries. $C A$ Cancer J Clin, 68, 394-424.

Cataldo VD, Gibbons DL, Pérez-Soler R, et al (2011). Treatment of non-small-cell lung cancer with erlotinib or gefitinib. N Engl J Med, 364, 947-55.

Cote ML, Haddad R, Edwards DJ, et al (2011). Frequency and type of epidermal growth factor receptor mutations in African Americans with non-small cell lung cancer. J Thorac
Oncol, 6, 627-30.

Dearden S, Stevens J, Wu YL, et al (2013). Mutation incidence and coincidence in non small-cell lung cancer: meta-analyses by ethnicity and histology (mutMap). Ann Oncol, 24, 2371-6.

Errihani H, Inrhaoun H, Boukir A, et al (2013). Frequency and type of epidermal growth factor receptor mutations in moroccan patients with lung adenocarcinoma. $J$ Thorac Oncol, 8, 1212-4.

Fakhruddin N, Mahfouz R, Farhat F, et al (2014). Epidermal growth factor receptor and KRAS mutations in lung adenocarcinoma: a retrospective study of the Lebanese population. Oncol Rep, 32, 2223-9.

Goldstraw P, Ball D, Jett JR, et al (2011). Non-small-cell lung cancer. Lancet, 378, 1727-40.

Izadian Z, Bahrami N, Naji T, et al (2017). Surveying the expression of CDH1 and EGFR genes in patients with anthracosis and its relationship with lung carcinoma. Asian Pac J Cancer Care, 2, 65-.

Jackman DM, Yeap BY, Sequist LV, et al (2006). Exon 19 deletion mutations of epidermal growth factor receptor are associated with prolonged survival in non-small cell lung cancer patients treated with gefitinib or erlotinib. Clin Cancer Res, 12, 3908-14.

Jazieh AR, Jaafar H, Jaloudi M, et al (2015). Patterns of epidermal growth factor receptor mutation in non-small-cell lung cancers in the Gulf region. Mol Clin Oncol, 3, 1371-4.

Kota R, Gundeti S, Gullipalli M, et al (2015). Prevalence and outcome of epidermal growth factor receptor mutations in non-squamous non-small cell lung cancer patients. Lung India, 32, 561-5.

Leary AF, Castro DG, Nicholson AG, et al (2012). Establishing an EGFR mutation screening service for non-small cell lung cancer - sample quality criteria and candidate histological predictors. Eur J Cancer, 48, 61-7.

Lee SH, Kim WS, Choi YD, et al (2015). Analysis of mutations in epidermal growth factor receptor gene in Korean patients with non-small cell lung cancer: Summary of a Nationwide Survey. J Pathol Transl Med, 49, 481-8.

Li M, Zhang Q, Liu L, et al (2011). The different clinical significance of EGFR mutations in exon 19 and 21 in nonsmall cell lung cancer patients of China. Neoplasma, $\mathbf{5 8}$, 74-81.

Liam CK, Wahid MI, Rajadurai P, et al (2013). Epidermal growth factor receptor mutations in lung adenocarcinoma in Malaysian patients. $J$ Thorac Oncol, 8, 766-72.

Liu Y, Ren Z, Wang J, et al (2016). Epidermal growth factor receptor-tyrosine kinase inhibitor therapy is especially beneficial to patients with exon 19 deletion compared with exon 21 L858R mutation in non-small-cell lung cancer: Systematic review and meta analysis. Thorac Cancer, 7, 406-14.

Midha A, Dearden S, McCormack R (2015). EGFR mutation incidence in non-small-cell lung cancer of adenocarcinoma histology: a systematic review and global map by ethnicity (mutMapII). Am J Cancer Res, 5, 2892-911.

Miltiadou K, Kalantzis I, Paraskeva M, et al (2020). Advanced lung cancer survival in times of economic hardship: A Greek Paradigm. Asian Pac J Cancer Care, 5, 19-25.

Naderi S, Ghorra C, Haddad F, et al (2015). EGFR mutation status in Middle Eastern patients with non-squamous non-small cell lung carcinoma: A single institution experience. Cancer Epidemiol, 39, 1099-102.

Palacio S, Pontes L, Prado E, et al (2019). EGFR mutation testing: Changing Patterns of Molecular Testing in Brazil. Oncologist, 24, 137-41.

Reinersman JM, Johnson ML, Riely GJ, et al (2011). Frequency of EGFR and KRAS mutations in lung adenocarcinomas in 
African Americans. J Thorac Oncol, 6, 28-31.

Shi Y, Au JS-K, Thongprasert S, et al (2014). A prospective, molecular epidemiology study of EGFR mutations in Asian patients with advanced non-small-cell lung cancer of adenocarcinoma histology (PIONEER). J Thorac Oncol, 9, 154-62.

Shi Y, Li J, Zhang S, et al (2015). Molecular epidemiology of EGFR mutations in Asian patients with advanced non-smallcell lung cancer of adenocarcinoma histology - Mainland China subset analysis of the PIONEER study. PLoS One, 10, 0143515 .

Szumera-Cieckiewicz A, Olszewski WT, Tysarowski A, et al (2013). EGFR mutation testing on cytological and histological samples in non-small cell lung cancer: a Polish, single institution study and systematic review of European incidence. Int J Clin Exp Pathol, 6, 2800-12.

Tfayli A, Fakhri G, Al Assaad M (2019). Prevalence of the epidermal growth factor receptor mutations in lung adenocarcinoma patients from the Middle East region. Ann Thorac Med, 14, 173-8.

Tfayli A, Rafei H, Mina A, et al (2017). Prevalence of EGFR and ALK mutations in lung adenocarcinomas in the Levant Area-a prospective analysis. Asian Pac J Cancer Prev, 18, 107.

Unal OU, Oztop I, Calibasi G, et al (2013). Relationship between epidermal growth factor receptor gene mutations and clinicopathological features in patients with non-small cell lung cancer in western Turkey. Asian Pac J Cancer Prev, 14, 3705-9.

Yatabe Y, Kerr KM, Utomo A, et al (2015). EGFR mutation testing practices within the Asia Pacific region: results of a multicenter diagnostic survey. $J$ Thorac Oncol, 10, 438-45.

Zaki MA, Ramadan RAEK, Mahmoud MI, et al (2015). Nonenriched PCR versus mutant-enriched PCR in detecting selected epidermal growth factor receptor gene mutations among nonsmall-cell lung cancer patients. Genet Test Mol Biomarkers, 19, 444-9.

Zhang YL, Yuan JQ, Wang KF, et al (2016). The prevalence of EGFR mutation in patients with non-small cell lung cancer: a systematic review and meta-analysis. Oncotarget, 7, 78985-93.

Zhou W, Christiani DC (2011). East meets West: ethnic differences in epidemiology and clinical behaviors of lung cancer between East Asians and Caucasians. Chin J Cancer, 30, 287-92.

Zhou Y, Yang Y, Yang C, et al (2017). Epidermal growth factor receptor (EGFR) mutations in non-small cell lung cancer (NSCLC) of Yunnan in southwestern China. Oncotarget, 8, 15023-33.

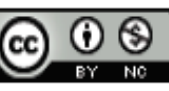

This work is licensed under a Creative Commons AttributionNon Commercial 4.0 International License. 